\title{
Opportunistic use of Spectral Holes in Karachi using Convolutional Neural Networks
}

\author{
Aamir Zeb Shaikh ${ }^{1}$, Shabbar Naqvi ${ }^{2}$, Minaal Ali ${ }^{3}$, Yamna Iqbal ${ }^{4}$, Abdul Rahim $^{5}$, Saima Khadim ${ }^{6}$, Talat Altaf ${ }^{7}$ \\ Department of Electronic Engineering, NED University of Engineering and Technology Karachi, Pakistan 1, 3, 4,5 \\ Department of Computer Systems Engineering, Balochistan University of Engineering and Technology Khuzdar, Pakistan ${ }^{2}$ \\ Department of Telecommunications Engg, Dawood University of Engineering and Technology, Karachi. Pakistan ${ }^{6}$ \\ Department of Electrical Engineering, Sir Syed University of Engineering and Technology Karachi, Pakistan ${ }^{7}$
}

\begin{abstract}
Wireless services appearing in the next generation wireless standard i.e. 6G include Internet of Everything (IoE), Holographic communications, smart transportation and smart cities require exponential rise in the bandwidth in addition to other requirements. The current static spectrum allocation policy does not allow any new entrant to exploit already grid-locked Radio Frequency (RF) spectrum. Hence, quest for larger bandwidth can be fulfilled through other technologies. These include exploiting sub-Terahertz band, Visible Light Communication and Cognitive Radio scheme or exploiting of RF bands in opportunistic fashion. Cognitive Radio is one of those engines to exploit the RF spectrum in secondary style. Cognitive Radio can use artificial intelligence driven algorithms to complete the task. Several intelligent algorithms can be used for better forecasting of spectral holes. Convolutional Neural Network (CNN) is a Deep Learning algorithm that can be used to predict the presence of a spectral hole that can be opportunistically exploited for efficient utilization of RF spectrum in secondary fashion. This paper investigates the performance of CNN for metropolitan Karachi city of Pakistan so that the users can be provided with uninterrupted access to the network even under busy hours. Dataset for the proposed setup is collected for $1805 \mathrm{MHz}$ frequency band through NI 2901 Universal Software Radio Peripheral (USRP) devices. The root mean square error (RMSE) for the predicted results using CNN appears to be 81.02 at epoch of 200 and mini-batch loss of 3281.8 . Based on the predicted results, it was concluded that CNN can be useful for investigating the possible opportunistic usage of RF spectrum; however, further investigation is required with different datasets.
\end{abstract}

Keywords-Cognitive radio; spectral hole; deep learning; Convolutional neural network (CNN)

\section{INTRODUCTION}

6G wireless communication standard and services discussion and investigation is already initiated. The proposed network initiatives promises to provide traffic capacity in the range of $1-10 \mathrm{Gbps} / \mathrm{m} 3$ as compared to $10 \mathrm{Gbps} / \mathrm{m} 3$ availability in $5 \mathrm{G}$ networks. It is assumed that the technologies that will play the role of enablers may include sub-Terahertz, visible light communication and Artificial Intelligence enabled cognitive communications. Additionally, greater use of multiRAT and multi-link schemes will also be required to rectify the issues arising from higher frequency propagation and providing higher reliability communication links [1].
The higher capacity requirement of the future technologies is a great challenge towards ubiquitous connectivity of wireless devices. Cognitive Radio is a possible option to play key role towards successful exploitation of Radio Frequency (RF) spectrum in opportunistic fashion. Thus, producing enough bandwidth to provide connectivity to wireless devices even in the congestion time. Cognitive Radio is a novel concept to incorporate artificial intelligence enabled techniques to exploit spectrum in opportunistic fashion. The major requirement towards a successful exploitation of RF band is to use primary bands in such a fashion that the usage does not produce harmful interference to the primary users. The cognitive radio is implemented through cognitive cycle [2]. The process of cognitive cycle starts with gathering RF spectrum monitoring [3]-[5]. This can be done through spectrum sensing process [6] and geo-location databases [7]. Spectrum monitoring combined with Radio Environmental Maps (REM) can also produce a better method of identifying unused Spectral bands [8]. Additionally, in [8] it is presented that the geo-location based database scheme typically results in an underutilized spectrum sharing scenario hence, better spectrum measurement scheme shall be incorporated that includes joint spectrum monitoring network and REM schemes[8] [9].

In the Artificial Intelligence enabled spectrum sensing radios, radios measure the Signal to Noise Ratio (SNR) and Energy levels of the primary radio transmission and this data is fed to the training algorithms. These algorithms predict the future nature of the RF spectrum bands regarding their usage in future i.e. empty or occupied. For this activity, different algorithms can be involved. As the spectrum assignment is a completely random assignment so a linear algorithm like Logistic regression may not produce useful results in all the channel conditions with different available active and secondary users [10]. While in most of the nonlinear environments, different algorithms use many other algorithms due to their specific use such as Support Vector Machines, KNearest Neighbor (KNN), Linear discriminant analysis (LDA) and Decision Trees [11]. In comparison to the aforementioned machine learning algorithms [12]-[14], deep learning algorithms typically perform much better.

The efficiency of Deep Learning algorithms is far better than the machine learning algorithms in general. However, this efficiency is resulted on the basis of available dataset and the nonlinear nature of the outputs. For example in case of linear functions, the logistic regression is preferred over the nonlinear 
algorithms because not only the regression produces simpler results but also predicts the given function in perfect fashion. However, when the available function is nonlinear and we have luxury to collect the 5000 samples of the data set we choose deep learning based algorithms in comparison to machine learning algorithms. Typical applications of deep learning algorithms include the real time test cases when the logistic regression don't produce the required level of accuracy. Additionally, there are complex patterns that can only better identified through these complex algorithms. Typically, audio and video data come into the picture of the said category [15].

Convolutional Neural Networks (CNNs) find their way to the area of deep learning based problems at enormously higher rate. These algorithms find their applications into the domain of machine translation, sentence classification, and sentiment analysis. Similar to the classic case of slide window algorithms, these algorithms slide over the given sequence of characters [15].

In the current work, $\mathrm{CNN}$ is chosen to predict the data for the opportunistic use of spectral holes in a large metropolitan city of Karachi Pakistan. For this purpose, a dataset is trained using RMSprop, which is a fast learning algorithm. The CNN has been used as a high performance classifier. The operation of convolution strengthens and reduces interference with the original signal features and have better tolerance to noise. The training parameters in $\mathrm{CNN}$ are less than in a fully connected network [16]. Two factors including Root Mean Square Error (RMSE) and loss function have been used to interpret the results.

The rest of the paper is divided in such a way that SectionII describes the related work. It is followed by Section-III which provides details of the proposed system. Section-IV explains the results obtained. Section-V is the last section providing Conclusion and future directions.

\section{RELATED WORK}

In the literature, Deep Neural Network based prediction algorithms are used by various authors to produce the data of spectral holes so that maximum number of users could be accommodated in available RF spectral bands [17]. Authors in [8] have presented a novel framework for spectrum monitoring purpose. The authors add CNN based algorithm to the spectrum measurement devices i.e. spectrum detectors that help the devices to identify the presence or absence of primary user signals (in this case, Radar signals are considered as primary users) even if the signals are found overlapped with other secondary users (in this case, WLAN and LTE are considered as secondary users). Furthermore, a large dataset containing various signal waveforms such as Radar pulses, LTE, WLAN and thermal noise waveforms is also developed that can be utilized by other researchers. A novel pre-processing scheme is also implemented that produces samples of amplitudes and phase shift of the collected waveforms. The results produced by the proposed scheme are excellent i.e. $99.6 \%$ accuracy is achieved when the proposed algorithm is run on testing dataset. This is in addition to the robustness of the proposed algorithm to noise. The proposed algorithm is also run on various SNR regimes that shows an improved performance of the selected algorithm in comparison to other models such as spectrogrambased CNN algorithms.

In [18], authors model the spectrum sensing issue as a classification problem by using CNN based processing. The received signal samples are initially normalized to overcome the effect of noise and uncertainties embedded in the received signal samples. The proposed model utilizes following different signaling schemes along with their variants i.e. PSK, FSK, QAM and AM. This encompasses reasonably wide variety of signals that are tested under the scenario considered in the paper. The maximum possible number of real world scenarios is considered for the training setup of proposed scheme. This feature will help the proposed scheme to perform better in the undefined wireless environments such as realworld wireless channels. The performance of proposed model is also compared with two algorithms maximum-minimum Eigen Value Ratio and frequency-domain entropy based methods. The results show better performance against its competitors even in the presence of colored noise. That shows the proposed scheme may find better use in the real-world environments. Additionally, to tackle the real world wireless issues, the proposed schemes is added with transfer learning technique to improve the performance. The data utilized in the proposed scheme is simulated except the experimentation into the real-world wireless environment testing.

Detection of RF signals under low SNR regime poses a great problem towards successful utilization of RF spectrum by opportunistic users [19]. In [16], authors attempt to solve the pressing issue (of detection under low SNR) by using CNN algorithm. The data collection for the proposed experimentation is performed by using Cyclostationary feature detector (An algorithm that converts the received signal into its signatures) and energy detection (An algorithm that measures the energy of the received signals). The performance of the proposed scheme is also compared with classic cyclostationary feature based detection schemes. The results show an improvement.

In classic spectrum sensing algorithms, typically model based approach is applied to sense the wireless environment i.e. energy and other methods, however, to devise better results the authors in [20] recommend a data driven approach to solve the problem of spectrum sensing through Deep convolutional network based spectrum monitoring approach. The proposed algorithm uses data to train itself. The sample covariance matrix is used as an input to the CNN algorithm. Maximum Aposterior Probability (MAP) based technique is used to devise the cost function. In offline method, the results of the proposed scheme show excellent performance by showing improved performance even under the correlated samples of primary user with correlation coefficient of 0.7 and uncorrelated primary user. Additionally, the proposed scheme works well than the conventional scheme i.e. Eigen value detection algorithm by approximately 7.5 times at an SNR level of $-14 \mathrm{~dB}$.

$\mathrm{CNN}$ is a branch of deep neural network in deep learning which is becoming popular tool for analyzing visual images. It includes image recognition, classification, and detection tasks [21]. They are referred to as convolutional neural networks 
because they use mathematical operation of convolution which is a specific type of linear operation [22]. In terms of Neural Networks, CNNs fall under category of generalized multilayer perceptron (MLPs). The major difference between CNN and MLP is that in MLP each input element is connected to each neuron in the hidden layer causing full connectivity whereas in CNN only a limited number of input elements called the receptive field is connected to only part of the hidden layer. $\mathrm{CNN}$ reduces the risk of over fitting of data in this way as compared to MLPs [23].

$\mathrm{CNN}$ is a type of deep learning which has been found to be very successful in areas of objects and image recognition, detection and segmentation challenges. CNNs applications in the various fields are on the rise. A number of reviews on applications of $\mathrm{CNN}$ have been done in the literature. A few recent reviews are shown in Table I. It can be seen that CNN applications are found in health informatics and in other image classification schemes.

A generalized $\mathrm{CNN}$ consists of input layer, an output layer and a number of hidden layers [8]. A generic $\mathrm{CNN}$ architecture has been shown in Fig. 1. CNN algorithm uses three layers to convert an input signal to output. The layers are: input, feature extraction e.g. learning and output layer. The input layer passes the data to a series of kernels i.e. convolutional layers with filters, pooling and fully connected (FC) layers. Then the Softmax function is used to decide the output data with a value of probability. Thus, it can be deduced that the convolutional layers works as an engine to extract features of input signal.

TABLE. I. YEAR WISE EXAMPLES OF RECENT REVIEWS DONE ON CNN

\begin{tabular}{|l|l|l|}
\hline S.No & Paper Title & $\begin{array}{l}\text { Year of } \\
\text { Publication }\end{array}$ \\
\hline 1 & $\begin{array}{l}\text { Deep convolutional neural networks for brain image } \\
\text { analysis on magnetic } \\
\text { resonance imaging: a review [24] }\end{array}$ & 2019 \\
\hline 2 & $\begin{array}{l}\text { Learning image-based spatial transformations via } \\
\text { convolutional neural networks: A review [25] }\end{array}$ & 2019 \\
\hline 3 & $\begin{array}{l}\text { Medical Image Analysis using Convolutional Neural } \\
\text { Networks: A Review [26] }\end{array}$ & 2018 \\
\hline 4 & $\begin{array}{l}\text { Skin Cancer Classification Using Convolutional } \\
\text { Neural Networks: systematic review [27] }\end{array}$ & 2018 \\
\hline 5 & $\begin{array}{l}\text { Deep Convolutional Neural Networks for Image } \\
\text { Classification: A Comprehensive Review [21] }\end{array}$ & 2017 \\
\hline 6 & $\begin{array}{l}\text { Convolutional Neural Networks for Inverse Problems } \\
\text { in Imaging: A Review [28] }\end{array}$ & 2017 \\
\hline
\end{tabular}

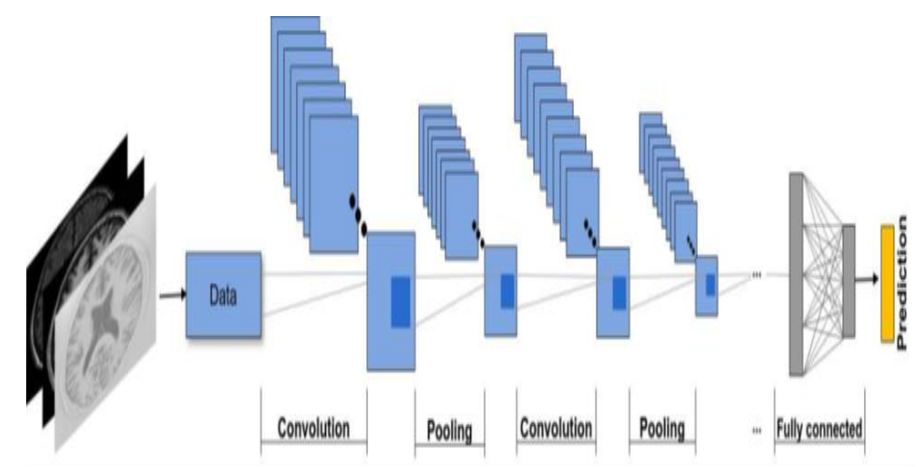

Fig. 1. Generic Architecture of Convolutional Neural Network [24].
It is also represented through mathematical operation of convolution. Convolutional layer works in conjunction with ReLU function. It is also called Rectified Linear Unit. There are many nonlinear functions that can be utilized such as Sigmoid; however, performance of ReLU under real environments is better than its competitors. The output of the layer is connected with pooling function. Pooling function is used to reduce the layer number of input parameters. Hence, it is also known as subsampling or down sampling unit. It is used to reduce the dimensionality of the input map; however, the significant information is always retained to make it highly useful function of the defined algorithm. It is generally implemented through max, average and sum pooling.

In this paper, $\mathrm{CNN}$ is used to predict the RF Usage in Karachi so that the available bands can be identified for further opportunistic usage. For this purpose, a dataset is also developed by using NI 2901 Universal Software Radio Peripheral (USRP) devices. CNN is applied to this dataset for prediction of the available RF bands. The RF band selected for the purpose is $1805 \mathrm{MHz}$. The RMSE for the proposed case comes out to be 81.02 at 200 epoch. The next section explains the details of the experimentation and analysis of the simulation results.

\section{PROPOSED SYSTEM}

Fig. 2 shows the structure of proposed system. In system, NI USRP 2901 is used to sense the presence of useful signals under real channel. A dataset is s developed using spectrum sensing algorithm. The Labview graphs are ported onto the Excel and Matlab to train the given algorithm. RMSprop is used for training purpose for the selected CNN Algorithm. Performance of RMSprop is closer to Momentum Gradient Descent Algorithm. It is used to control the learning rate thus taking larger steps produces convergence more quickly in horizontal direction. Two parameters including RMSE and Loss Function were used for investigation of the results. RMSE can be defined as standard deviation of residuals (prediction errors).

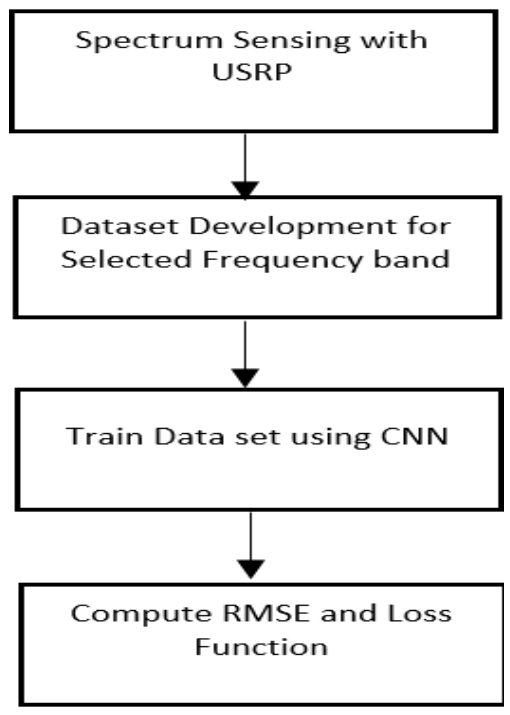

Fig. 2. Structure of Proposed System. 
In other words, it shows the difference between the regression line and spread of the prediction errors. Machines work typically through loss function parameter. Loss function is used to determine the output of the given algorithm and the given target value. However, under circumstances when predicted values deviate too much from loss functions, the estimation error algorithms uses optimization functions.

\section{RESULTS}

Fig. 3 shows two parameters for the proposed setup. These include RMSE and Loss function. Total time to generate the results from proposed setup is 4 minutes and 40 seconds. One iteration is assumed for each epoch. Total epochs and iterations are taken to be 200. For first epoch and iteration, time requirement is 1 second. Mini- batch RMSE is 287 min-batch loss comes out to be 41185.5 . Base Learning rate is 0.0010 . Learning rate is used to determine how quickly CNN model learns the model. For 200 epoch and iteration, time requirement is 4 minutes and 40 seconds, whereas mini-batch RMSE is 81.02 and Mini-batch Loss is 3281.8 and Base learning Rate is $5.4976 \mathrm{e}-31$. Thus, as the iterations are increased RMSE and Loss function decreases but also Base Learning Rate decreases in larger amount. It means that chances of reaching a global optimal solution are high but there is also risk of getting stuck at a sub optimal solution. The training statistics for the experiments conducted is also shown in Table II for 200 iterations as till that time the result becomes consistent in terms of RMSE and loss function. Results indicate that for the dataset of Karachi, after few iterations, RMSE and Loss function become consistent, however rapid decrease in learning rate is an area which needs to be further investigated. It is worth mentioning that the proposed setup is implemented using a single CPU. Hence, for large amount of data, GPUs are recommended to use.

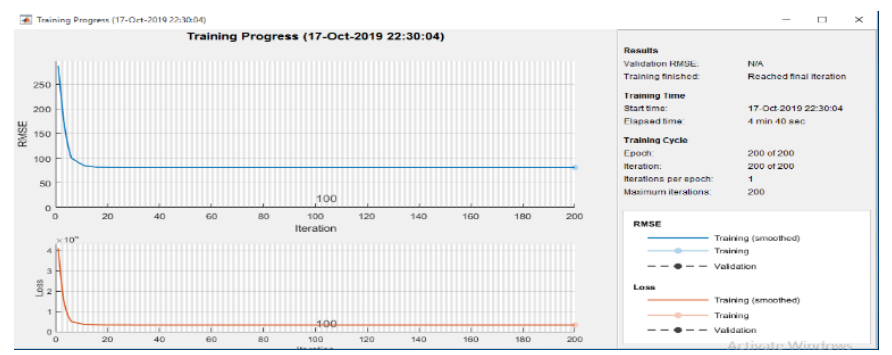

Fig. 3. Shows RMSE and Loss Functions for the Proposed Algorithm.

TABLE. II. TRAINING STATISTICS FOR THE PROPOSED ALGORITHM

\begin{tabular}{|l|l|l|l|l|l|}
\hline EPOCH & Iteration & $\begin{array}{l}\text { Time } \\
\text { Elapsed } \\
\text { (hh:mm:ss) }\end{array}$ & $\begin{array}{l}\text { Mini } \\
\text { Batch } \\
\text { RMSE }\end{array}$ & $\begin{array}{l}\text { Mini } \\
\text { Batch } \\
\text { LOSS }\end{array}$ & $\begin{array}{l}\text { Base } \\
\text { Learning } \\
\text { Rate }\end{array}$ \\
\hline 1 & 1 & $00: 00: 01$ & 287.00 & 41185.5 & 0.0010 \\
\hline 50 & 50 & $00: 01: 15$ & 81.02 & 3281.8 & $5.1200 \mathrm{e}-10$ \\
\hline 100 & 100 & $00: 02: 24$ & 81.02 & 3281.8 & $5.2429 \mathrm{e}-17$ \\
\hline 150 & 150 & $00: 03: 32$ & 81.02 & 3281.8 & $5.3678 \mathrm{e}-24$ \\
\hline 200 & 200 & $00: 04: 40$ & 81.02 & 3281.8 & $5.4976 \mathrm{e}-31$ \\
\hline
\end{tabular}

\section{CONCLUSION AND FUtURE WORK}

In this work, deep learning using $\mathrm{CNN}$ has been used to predict the spectral spaces for Karachi city. The algorithm takes longer time to train as compared to machine learning algorithms. The proposed setup is implemented using a single CPU machine. The results show that the best possible RMSE and Loss function are achieved at $50^{\text {th }}$ iterations. Even after increasing the iterations, no significant progress on these parameters is found. However, the Base Learning Rate decreases significantly after running the machine for 200 iterations and needs to be further investigated and compared with other deep learning algorithms. This work was based on data set for metropolitan city of Karachi. In future, we aim at investigating the rural area datasets to find out any significant differences while changing datasets with $\mathrm{CNN}$ and other deep learning methods for investigating opportunistic use of spectral holes with the aid of artificial intelligence techniques.

\section{ACKNOWLEDGMENT}

The authors would like to thank the Administration of NED University of Engineering \& Technology, Karachi, Pakistan, for providing resources to complete this research.

\section{REFERENCES}

[1] E. C. Strinati, S. Barbarossa, J. L. Gonzalez-Jimenez, D. Kténas, N. Cassiau, and C. Dehos, “6G: The Next Frontier,” pp. 1-16, 2019.

[2] N. Savage, “Cognitive radio," Technol. Rev., vol. 109, no. 1, pp. 61-62, 2006, doi: 10.15373/2249555x/apr2012/28.

[3] A. Z. Shaikh and T. Altaf, "Collaborative Spectrum Sensing under Suburban Environments,” Int. J. Adv. Comput. Sci. Appl., vol. 4, 2013.

[4] A. Z. Shaikh and L. Tamil, "Cognitive radio enabled telemedicine system," Wirel. Pers. Commun., vol. 83, no. 1, pp. 765-778, 2015.

[5] A. A. Khan, A. Z. Shaikh, S. Naqvi, and T. Altaf, "A Novel Cognitive Radio Enabled IoT System for Smart Irrigation,” J. Inform. Math. Sci., vol. 9, no. 1, pp. 129-136, 2017.

[6] A. Ahmed, A. Zeb, S. Naqvi, and T. Altaf, "Implementation of Cooperative Spectrum Sensing Algorithm using Raspberry Pi," Int. J. Adv. Comput. Sci. Appl., vol. 7, no. 12, pp. 363-367, 2016, doi: 10.14569/ijacsa.2016.071247.

[7] H. Yilmaz Birkan, T. Tugcu, F. Alagöz, and S. Bayhan, "Radio environment map as enabler for practical cognitive radio networks," IEEE Commun. Mag., vol. 51, no. 12, pp. 162-169, 2013, doi: 10.1109/MCOM.2013.6685772.

[8] A. Selim, F. Paisana, J. A. Arokkiam, Y. Zhang, L. Doyle, and L. A. DaSilva, "Spectrum Monitoring for Radar Bands Using Deep Convolutional Neural Networks," 2017 IEEE Glob. Commun. Conf. GLOBECOM 2017 - Proc., vol. 2018-Janua, pp. 1-6, 2017, doi: 10.1109/GLOCOM.2017.8254105.

[9] L. Gavrilovska et al., "Enabling LTE in TVWS with radio environment maps: From an architecture design towards a system level prototype," Comput. Commun., vol. 53, pp. 62-72, 2014, doi: 10.1016/j.comcom.2014.07.008.

[10] M. Gail, K. Krickeberg, J. M. Samet, A. Tsiatis, and W. Wong, Statistics for Biology and Health Series Editors. 2010.

[11] G. Biagetti, P. Crippa, L. Falaschetti, G. Tanoni, and C. Turchetti, "A comparative study of machine learning algorithms for physiological signal classification," Procedia Comput. Sci., vol. 126, pp. 1977-1984, 2018, doi: 10.1016/j.procS.2018.07.255.

[12] S. Khadim, A. Waqar, A. Zeb, I. Khan, and I. Hussain, "Smart Cognitive Cellular Network," Int. J. Future Gener. Commun. Netw., vol. 10, no. 12, pp. 23-34, 2017. 
[13] A. Waqar, S. Khadim, A. Zeb, S. Amir, and I. Khan, "A Survey on Cognitive Radio Network using Artificial Neural Network,” Int. J. Future Gener. Commun. Netw., vol. 10, no. 11, pp. 11-18, 2017.

[14] I. Khan, S. Wasi, A. Waqar, and S. Khadim, "Comparative analysis of ANN techniques for predicting channel frequencies in cognitive radio," Int. J. Adv. Comput. Sci. Appl., vol. 8, pp. 296-303, 2017.

[15] D. Learning, 技術者が知っておきたい Deep Learning の基礎と 組込 みでの利用 〜 今さら聞いてください Deep Learning 〜, vol. 26, no. 7553. 2016

[16] D. Han et al., "Spectrum sensing for cognitive radio based on convolution neural network," Proc. - 2017 10th Int. Congr. Image Signal Process. Biomed. Eng. Inform. CISP-BMEI 2017, vol. 2018-Janua, pp. 1-6, 2018, doi: 10.1109/CISP-BMEI.2017.8302117.

[17] M. A. Khan, A. Z. Shaikh, S. Naqvi, S. Khadim, and T. Altaf, "Deep Learning Enabled Spectrum Sensing Radio for Opportunistic Usage," IJCSNS, vol. 19, no. 11, p. 179, 2019.

[18] S. Zheng, S. Chen, P. Qi, H. Zhou, and X. Yang, "Spectrum Sensing Based on Deep Learning Classification for Cognitive Radios," pp. 1-7, 2019.

[19] A. Sahai, N. Hoven, and R. Tandra, "Some Fundamental Limits on Cognitive Radio," Allerton Conf. Control Commun. Comput., pp. 16621671, 2004, doi: 10.1.1.123.5645.

[20] C. Liu, J. Wang, X. Liu, and Y. C. Liang, "Deep CM-CNN for Spectrum Sensing in Cognitive Radio," IEEE J. Sel. Areas Commun., vol. 37, no. 10, pp. 2306-2321, 2019, doi: 10.1109/JSAC.2019.2933892.
[21] W. Rawat and Z. Wang, Deep convolutional neural networks for image classification: A comprehensive review, Neural computing. MIT Press Journals, 2017.

[22] I. Goodfellow, Y. Bengio, and A. Courville, Deep learning. MIT press, 2016.

[23] A. Botalb, M. Moinuddin, U. M. Al-Saggaf, and S. S. Ali, "Contrasting Convolutional Neural Network (CNN) with Multi-Layer Perceptron (MLP) for Big Data Analysis," in 2018 International Conference on Intelligent and Advanced System (ICIAS), 2018, pp. 1-5.

[24] J. Bernal et al., "Deep convolutional neural networks for brain image analysis on magnetic resonance imaging: a review," Artif. Intell. Med., vol. 95, pp. 64-81, 2019.

[25] N. J. Tustison, B. B. Avants, and J. C. Gee, "Learning image-based spatial transformations via convolutional neural networks: a review," Magn. Reson. Imaging, 2019.

[26] S. M. Anwar, M. Majid, A. Qayyum, M. Awais, M. Alnowami, and M. K. Khan, "Medical image analysis using convolutional neural networks: a review," J. Med. Syst., vol. 42, no. 11, p. 226, 2018.

[27] T. J. Brinker et al., "Skin cancer classification using convolutional neural networks: systematic review," J. Med. Internet Res., vol. 20, no. 10, p. e11936, 2018.

[28] M. T. McCann, K. H. Jin, and M. Unser, "Convolutional neural networks for inverse problems in imaging: A review," IEEE Signal Process. Mag., vol. 34, no. 6, pp. 85-95, 2017. 